University of Nebraska - Lincoln

DigitalCommons@University of Nebraska - Lincoln

9-28-1992

\title{
Competition between ponderomotive and thermal forces in short- scale-length laser plasmas
}

X. Liu

University of Michigan, Ann Arbor

Donald P. Umstadter

University of Nebraska-Lincoln, donald.umstadter@unl.edu

Follow this and additional works at: https://digitalcommons.unl.edu/physicsumstadter

Part of the Physics Commons

Liu, X. and Umstadter, Donald P., "Competition between ponderomotive and thermal forces in short-scalelength laser plasmas" (1992). Donald Umstadter Publications. 34.

https://digitalcommons.unl.edu/physicsumstadter/34

This Article is brought to you for free and open access by the Research Papers in Physics and Astronomy at DigitalCommons@University of Nebraska - Lincoln. It has been accepted for inclusion in Donald Umstadter Publications by an authorized administrator of DigitalCommons@University of Nebraska - Lincoln. 


\title{
Nonlinear Plasma Waves Resonantly Driven by Optimized Laser Pulse Trains
}

\author{
D. Umstadter, ${ }^{1}$ E. Esarey, ${ }^{2}$ and J. Kim ${ }^{1}$ \\ ${ }^{1}$ Center for Ultrafast Optical Science, University of Michigan, Ann Arbor, Michigan 48109 \\ ${ }^{2}$ Beam Physics Branch, Plasma Physics Division, Naval Research Laboratory, Washington, D.C. 20375
}

(Received 22 October 1993)

\begin{abstract}
A method for generating large-amplitude plasma waves, which utilizes an optimized train of independently adjustable, intense laser pulses, is discussed and analyzed. Both the pulse widths and interpulse spacings are optimally determined such that resonance is maintained and the plasma wave amplitude is maximized. By mitigating the effects of both phase and resonant detuning, and by reducing laser-plasma instabilities, the use of appropriately tailored multiple laser pulses is a highly advantageous technique for accelerating electrons. Practical methods of producing the required pulse trains are suggested.

PACS numbers: $52.75 . \mathrm{Di}, 52.35 . \mathrm{Mw}, 52.40 . \mathrm{Nk}$
\end{abstract}

The generation of large-amplitude, relativistic plasma waves is a subject of much current interest because of its potential use for ultrahigh-gradient electron acceleration [1-4]. While conventional rf-driven accelerators are limited to fields $\leq 1 \mathrm{MV} / \mathrm{cm}$, plasma accelerators have been shown experimentally to support gradients $\leq 100 \mathrm{MV} / \mathrm{cm}$ [4]. The maximum axial electric field of a relativistic plasma wave, as predicted by $1 \mathrm{D}$ cold fluid theory, is the "wave-breaking" field [5], $E_{\mathrm{WB}}=\left(m_{e} \alpha \omega_{p} / e\right) \sqrt{2\left(\gamma_{p}-1\right)}$, which can exceed 1 $\mathrm{GV} / \mathrm{cm}$, where $\omega_{p}=\left(4 \pi e^{2} n_{e 0} / m_{e}\right)^{1 / 2}$ is the electron plasma frequency, $n_{e 0}$ is the ambient electron density, $\gamma_{p}=\left(1-v_{p}^{2} / c^{2}\right)^{-1 / 2}$, and $v_{p}$ is the phase velocity.

Two major types of laser-driven, plasma-based accelerators have been proposed: the plasma beat-wave accelerator (PBWA) [2] and the laser wake-field accelerator (LWFA) $[2,3]$. In the PBWA, two laser beams of frequencies $\omega$ and $\omega-\omega_{p}$ are optically mixed in a plasma to produce a laser beat wave, in effect a train of fixed equally spaced pulses of equal pulse widths. In the LWFA, a single, ultrashort, and intense laser pulse [6] drives a plasma wave "wake field." In both cases, the maximum plasma-wave amplitude results when the pulse spacing and/or pulse width $\tau$ are "resonant" with the plasma, or $\tau \sim 2 \pi / \omega_{p}$. When a plasma wave grows to large amplitude, however, it becomes nonlinear and both its resonance width and period change. At this point, these schemes begin to lose their efficiency because the laser pulse widths and interpulse spacings do not change accordingly, known in the latter case as resonant detuning.

Recently, the self-modulated LWFA has been suggested $[7,8]$. Here, a single laser pulse is incident on a plasma with a density that is higher than the "resonant density." Because of a self-modulation instability, the pulse breaks up into multiple pulses, each of which is resonant. Although higher plasma densities and the highintensity multiple-pulse structure lead to higher wakefield amplitudes, they are difficult to achieve simultaneously due to plasma defocusing [9].

Additionally, electron acceleration is limited at high $n_{e 0}$ by phase detuning, i.e., accelerated electrons (with $v \rightarrow c$ ) outrun the plasma wave (with $v_{p} \simeq v_{g}<c$ ). The maximum energy gain, $\Delta W_{\max }$, of an electron in a 1D sinusoidal plasma wave of amplitude $E_{z}=\epsilon E_{\mathrm{WB}}$ is $\Delta W_{\max } \simeq 4 e c \epsilon E_{\mathrm{WB}} \gamma_{p}^{2} / \omega_{p}$, where $\epsilon \leq 1$ is a constant. Since $\gamma_{p} \simeq \gamma_{g} \simeq \omega / \omega_{p} \gg 1, \Delta W_{\max } \simeq \epsilon m_{e} c^{2}\left(2 \gamma_{g}\right)^{5 / 2}$. For example, $\Delta W_{\max } \simeq 4.5 \mathrm{GeV}$, assuming a laser wavelength of $\lambda \simeq 2 \pi c / \omega=1 \mu \mathrm{m}, n_{e 0}=10^{18} \mathrm{~cm}^{-3}$ $\left(E_{\mathrm{WB}}=7.8 \mathrm{GV} / \mathrm{cm}\right)$ and $\epsilon=25 \%$. Hence, at the high densities required either for self-modulation or for the use of an ultrashort pulse in the standard LWFA, $\gamma_{g}$ is relatively low and acceleration is limited, $\Delta W \sim \epsilon n_{e 0}^{-5 / 4}$.

We propose an alternative accelerator concept, which we call the resonant laser-plasma accelerator (RLPA), that combines the virtues of these others, but has the following additional advantages: (i) By utilizing a train of laser pulses with independently adjustable pulse widths and interpulse spacings, which are varied in an optimized manner, resonance with both the changing plasma-wave period and resonance width can be maintained in the nonlinear regime, and the maximum plasma-wave amplitude is achieved; (ii) lower plasma densities can be used, thus avoiding electron-phase detuning; and (iii) lower peak laser intensities can be used, thus allowing for a reduction of laser-plasma instabilities. Berezhiani and Murusidze [10] considered a secondary pulse to enhance or diminish the wake field. Nakajima [11] considered using a ring resonator, with fixed pulse width and interpulse spacing. Unlike our treatment, however, neither of these past studies considered the effects of variation of the plasma-wave resonance widths on the optimal widths of realistic, finite rise-time laser pulses, and the resulting efficiency of both large-amplitude plasma wave generation and electron acceleration.

The laser-plasma interaction is modeled by the relativistic fluid Maxwell equations. The laser pulse is described by the normalized transverse vector potential, $\mathbf{a}=e \mathbf{A}_{\perp} / m_{e} c^{2}$. The laser envelope, $|a|$, is assumed to be nonevolving and a function of only $\zeta=z-v_{g} t$, where $v_{g}$ is the group velocity (assumed constant). Circular 
polarization is assumed, i.e., $a^{2}=a^{2}(\zeta)$. The plasma response is described by the normalized electrostatic potential, $\phi=e \Phi / m_{e} c^{2}$, which in the 1D limit obeys the nonlinear Poisson equation $[10,12]$

$$
\frac{d^{2}}{d \zeta^{2}} \phi=k_{p}^{2} \gamma_{g}^{2}\left[\beta_{g}\left(1-\frac{1+a^{2}}{\gamma_{g}^{2}(1+\phi)^{2}}\right)^{-1 / 2}-1\right] \text {, }
$$

where $\beta_{g}=v_{g} / c, \gamma_{g}=\left(1-\beta_{g}^{2}\right)^{-1 / 2}$, and $k_{p}=\omega_{p} / c$ is the plasma wave number. In deriving Eq. (1), $\phi$ was assumed to be a function of only $\zeta$, i.e., $v_{p} \simeq v_{g}$. In the limit $a^{2} \ll 1, \gamma_{g}=\omega / \omega_{p}$. As in all laser-plasma accelerators, a preformed density channel can be used to prevent pulse diffraction. Simulations indicate that an intense laser pulse can propagate within a channel over extended distances without significant distortions [7].

Several properties of the plasma wave can be determined analytically from Eq. (1) for a series of square laser pulses. For an optimal square pulse train, it can be shown that the amplitude of the wake behind the $n$th pulse oscillates between $x_{\min _{n}} \leq x \leq x_{\max _{n}}$, where

$$
\begin{gathered}
Y_{n} / \gamma_{g}^{2}=X_{n}\left(1+\beta_{g}^{2}\right)-2 \beta_{g}\left(X_{n}^{2}-1 / \gamma_{g}^{2}\right)^{1 / 2} \\
X_{n} / \gamma_{g}^{2}=Y_{n-1}\left(1+\beta_{g}^{2}\right)-2 \beta_{g}\left(Y_{n-1}^{2}-\gamma_{\perp_{n}}^{2} / \gamma_{g}^{2}\right)^{1 / 2} .
\end{gathered}
$$

Here, $x=1+\phi, \gamma_{\perp_{n}}=\left(1+a_{n}^{2}\right)^{1 / 2}, a_{n}$ is the amplitude of the $n$th pulse, $x_{\min _{0}} \equiv 1, X_{n} \equiv x_{\max _{n}}$, and $Y_{n} \equiv x_{\min _{n}}$. Furthermore, the maximum electric field behind the $n$th pulse is given by

$$
{X^{\prime}}_{n}^{2} / 2 k_{p}^{2} \gamma_{g}^{2}=X_{n}-1 / \gamma_{g}^{2}-\beta_{g}\left(X_{n}^{2}-1 / \gamma_{g}^{2}\right)^{1 / 2},
$$

where the axial electric field is related to $x^{\prime}$ (the prime denotes $d / d \zeta$ ) by $E_{z}=-m_{e} c^{2} x^{\prime} / e$. In deriving Eqs. (2)(4), the spacing between pulses and the pulse lengths are assumed to be optimized, such that the $n$th pulse begins at $x=x_{\min _{n-1}}$ and ends at $x=x_{\max _{n}}$. Wave breaking occurs when $x_{\min _{n}} \rightarrow 1 / \gamma_{g}$ (the point where the electron fluid velocity equals $v_{p}$ ) which implies $x_{\max _{n}} \rightarrow x_{\mathrm{WB}}=$ $\left(2 \gamma_{g}^{2}-1\right) / \gamma_{g}$. This corresponds to a wave-breaking electric field of $x^{\prime 2}{ }_{\mathrm{WB}}=2 k_{p}^{2}\left(\gamma_{g}-1\right)$, or $E_{z}=E_{\mathrm{WB}}$ [5].

Numerical solutions to Eq. (1) indicate that for $x^{2} \ll$ $x_{\mathrm{WB}}^{2}$, Eq. (1) can be approximated by the limit $\beta_{g} \rightarrow 1$, i.e., $2 x^{\prime \prime}=k_{p}^{2}\left(\gamma_{\perp}^{2} / x^{2}-1\right)$, where $\gamma_{\perp}^{2}=1+a^{2}$. For a series of square pulses, analytic solutions can also be readily obtained from this reduced equation. In particular, $x_{\min _{n}}=1 / x_{\max _{n}}, x_{\max _{n}}=\gamma_{\perp_{1}}^{2} \gamma_{\perp_{2}}^{2} \cdots \gamma_{\perp_{n}}^{2}$, and $x_{\max _{n}}^{\prime}=k_{p}\left(x_{\max _{n}}^{1 / 2}-x_{\max _{n}}^{-1 / 2}\right)$. Furthermore, the optimal width of the $n$th pulse, $L_{n}$, and the nonlinear wavelength of the wake behind the $n$th pulse, $\lambda_{N_{n}}$, are given by $k_{p} L_{n}=2 x_{\max _{n}}^{1 / 2} E\left(\rho_{n}\right)$ and $k_{p} \lambda_{N_{n}}=4 x_{\max _{n}}^{1 / 2} E\left(\hat{\rho}_{n}\right)$, where $E$ is the complete elliptic integral of the second kind, $\rho_{n}^{2}=1-\gamma_{\perp_{n}}^{2} x_{\max _{n}}^{-2}$ and $\hat{\rho}_{n}^{2}=1-x_{\max _{n}}^{-2}$. The optimal spacing between the end of the $n$th pulse and the $n$th +1 pulse is an integer multiple of $\lambda_{N_{n}}$. Note for equal pulse amplitudes, i.e., $a_{1}=a_{2}=\cdots \equiv a_{0}$,

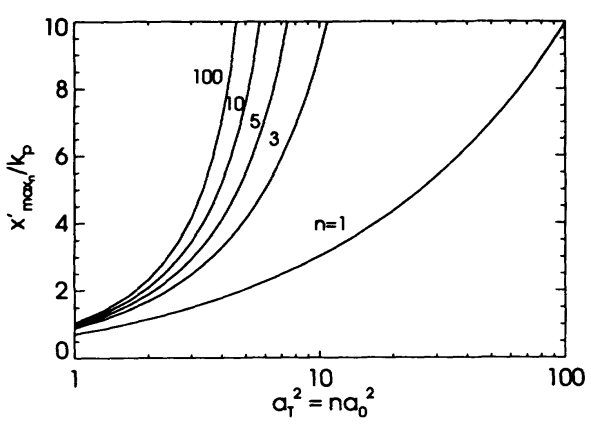

FIG. 1. The maximum electric field $\left(x_{\max _{n}}^{\prime} / k_{p}\right)$ vs the quantity $a_{T}^{2}=n a_{0}^{2}$ for $n=1,3,5,10$, and 100 .

$x_{\max _{n}}=\gamma_{\perp_{0}}^{2 n}=\left(1+a_{0}^{2}\right)^{n}$. In the limit $x_{\max _{n}}^{2} \gg 1$, $k_{p} L_{n} \simeq 2 \gamma_{\perp_{0}}^{n}, k_{p} \lambda_{N n} \simeq 4 \gamma_{\perp_{0}}^{n}$, and $x_{\max _{n}}^{\prime} \simeq k_{p} \gamma_{\perp_{0}}^{n}$.

The maximum normalized electric field, $x_{\max }^{\prime} / k_{p}$, for a train of $n$ square pulses of equal amplitudes, is plotted in Fig. 1 versus the quantity $a_{T}^{2}=n a_{0}^{2}$, using the results of Eqs. (2)-(4). For $\gamma_{g} \gg 1$ and $x^{2} \ll x_{\mathrm{WB}}^{2}, x_{\max }^{\prime} / k_{p}$ is approximately independent of $n_{e 0}$. The curves show the result for $1,3,5,10$, and 100 pulses. Figure 1 indicates that just a few optimized square pulses are far more efficient than a single pulse. For example, at $n_{e 0}=10^{15}$ $\mathrm{cm}^{-3}\left(\lambda=1 \mu \mathrm{m}, \gamma_{g} \simeq 10^{3}, E_{\mathrm{WB}} \simeq 1.4 \mathrm{GV} / \mathrm{cm}\right)$, three square pulses can be used with an intensity $I=2.7 \times 10^{18}$ $\mathrm{W} / \mathrm{cm}^{2}$ pulse $\left(a_{0}^{2}=1\right)$ and a total pulse train energy of $I \tau_{\text {tot }}=20 \mathrm{MJ} / \mathrm{cm}^{2}$ to produce $E_{z}=0.1 \mathrm{GV} / \mathrm{cm}$. Here, $\tau_{\text {tot }}$ is the sum of the pulse durations in the train and $2.7 a_{0}^{2} \simeq 10^{-18} \lambda^{2}[\mu \mathrm{m}] I\left[\mathrm{~W} / \mathrm{cm}^{2}\right]$. A single pulse at $n_{e 0}=10^{15} \mathrm{~cm}^{-3}$ requires $I=3 \times 10^{19} \mathrm{~W} / \mathrm{cm}^{2}\left(a_{0}^{2}=11\right)$, over an order of magnitude higher intensity than in each pulse in the train, and a total energy 6 times greater $\left(I \tau_{\text {tot }}=120 \mathrm{MJ} / \mathrm{cm}^{2}\right)$, to produce this same $E_{z}$. (A low density was chosen for this example so that finite rise time effects could be neglected, as discussed below.) The efficiency advantage of multiple pulses increases with increasing $n$ or $a_{0}^{2}$.

Figure 2(a) shows an example of an optimized square pulse train $\left(n=3, a_{0}=1, n_{e 0}=10^{15} \mathrm{~cm}^{-3}\right)$, as obtained by a numerical solution of Eq. (1), in which the widths and spacing between pulses are varied in order to maximize $x_{\max }$. The laser pulses are optimally located in the regions where $d \phi / d \zeta>0$. When a train that is not optimum is used, for instance fixed interpulse spacings (as in the case of the PBWA), $x_{\max }$ reaches some saturated value before being driven down by destructive interference when the pulses become out of phase with the wave, i.e., when they are located in regions where $d \phi / d \zeta<0$. The plasma wave is driven most effectively near $\phi=\phi_{\min }$ (where $n_{e}$ is maximum) and least effectively as $\phi \rightarrow \phi_{\max }$.

The above results have assumed square pulses with an infinitesimally short rise time. In practice, the rise time $\tau_{\text {rise }}$ of a pulse directly out of a laser is finite and determined by the bandwidth of the laser amplifiers, e.g., cur- 

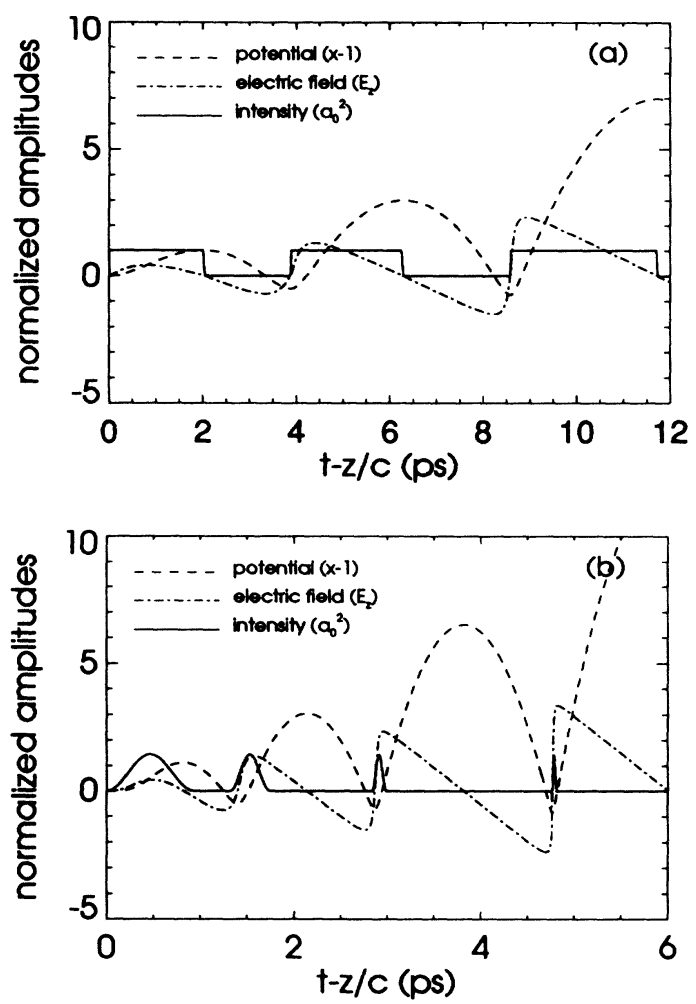

FIG. 2. Numerical solutions for optimized pulse trains: (a) square pulses at $n_{e}=10^{15} \mathrm{~cm}^{-3}$ with $a_{0} \simeq 1$, and (b) sine pulses at $n_{e}=10^{16} \mathrm{~cm}^{-3}$ with $a_{0}=1.2$.

rently, the minimum amplified pulse width is $\tau_{\min } \simeq 50$ fs. In the following, we will consider pulses with an envelope profile $a(\zeta)$ given by a half period of a sine function. (Gaussian profiles give qualitatively similar results.)

Figure 2(b) shows an example of a sine pulse train $\left(n=4, a_{0}=1.2, n_{e 0}=10^{16} \mathrm{~cm}^{-3}\right)$ that was optimized numerically. The first pulse in Fig. 2(b) has an optimum pulse width $\tau=\tau_{\mathrm{opt}}=940 \mathrm{fs}$ (resonant with $n_{e 0}=10^{16}$ $\mathrm{cm}^{-3}$ and $a_{0}=1.2$ ) and the final pulse has $\tau=\tau_{\mathrm{opt}}=$ $\tau_{\min } \simeq 50 \mathrm{fs}\left(I \tau_{\text {tot }}=5.3 \mathrm{MJ} / \mathrm{cm}^{2}\right)$, which gives $E_{z}=0.34$ $\mathrm{GV} / \mathrm{cm}(\epsilon=0.14)$. As in the square wave case, $\lambda_{N_{n}}$ and thus the spacing between pulses increases with each succeeding pulse as $x_{\max }$ increases.

The sensitivity of the growth of $x_{\max }^{\prime}$ to changes in the location of the final laser pulse of Fig. 2(b) was studied numerically. It is governed by both the number of pulses (decreasing with increasing $n$ ) and the $Q$ of the resonance (increasing with increasing $Q$ ), where $Q \sim x_{\max }$ is as defined below. Pulses with $\tau>\tau_{\mathrm{opt}}$ are found to be less sensitive to changes in interpulse spacing than those with $\tau=\tau_{\text {opt }}$, without sacrificing much efficiency. For instance, if the pulse width of the last pulse were $\tau=4 \tau_{\mathrm{opt}}=200 \mathrm{fs}$ (instead of $\tau_{\mathrm{opt}}$ ), it is found that a decrease in the optimal spacing between the last and the third pulse $\left(\lambda_{N_{3}}\right)$ by $25 \mathrm{fs}$ (corresponding to $\delta \lambda_{N_{3}} / c \tau_{\text {opt }}=50 \%$ ) results in a decrease of $E_{z}$ (from the

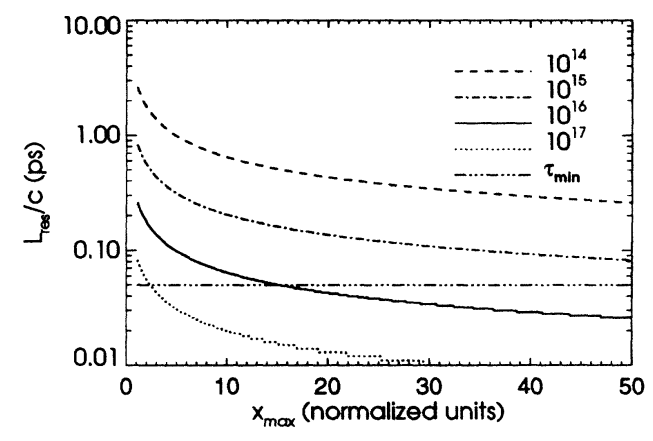

FIG. 3. Plot of $L_{\mathrm{res}} / c$ vs $\epsilon=E_{z} / E_{\mathrm{WB}}$ for various densities. Finite rise-time effects are important for $L_{\text {res }} / c<\tau_{\min }$.

value obtained using $\tau=\tau_{\mathrm{opt}}$ and the optimal position) by only $15 \%$ (instead of $30 \%$ ). Note, in the $\tau=4 \tau_{\text {opt }}$ case, $I \tau_{\text {tot }}=5.9 \mathrm{MJ} / \mathrm{cm}^{2}$, corresponding to an energy increase of only $11 \%$. The amplitude is less sensitive to an increase in the spacing, since this moves the pulse further from the $d \phi / d \zeta<0$ region, and thus the wake continues to be enhanced, but less effectively.

Note that whereas $\tau_{\mathrm{opt}} \sim \lambda_{N_{n}} / c$ for succeeding square wave pulses increases with increasing $x_{\max }$, the opposite is true for multiple sine pulses. This difference arises because, whereas for square pulses $\tau$ is independent of $\tau_{\text {rise }}$, for sine pulses $\tau \simeq 2 \tau_{\text {rise }}$. It is more advantageous to have a short sine pulse width $\left(\tau \ll \lambda_{N_{n}} / c\right)$, so that the highest pulse amplitude is reached near $\phi_{\min }$ (where it is most effective in driving the plasma wave), than to have a long sine pulse width $\left(\tau \simeq \lambda_{N_{n}} / c\right)$, so that the pulse is driving the wave for a longer time, albeit mostly when it is less effective (away from $\phi_{\min }$ ). Sine pulses are found to be more effective than square pulses for this same reason. For the later sine pulses, $\tau_{\text {opt }}$ is found to be approximately given by the width of the region where $\phi<0$ and $d \phi / d \zeta>0$, which defines a "resonance width" $L_{\text {res }}$. For the wake behind the $n$th pulse, $L_{\text {res }}$ can be determined from Eq. (1) in the limit $v_{g}=c$,

$$
k_{p} L_{\mathrm{res}}=2 x_{\max _{n}}^{1 / 2}\left|E\left(\pi / 2, \hat{\rho}_{n}\right)-E\left(\alpha_{1}, \hat{\rho}_{n}\right)\right|,
$$

where $\hat{\rho}_{n}^{2} \sin ^{2} \alpha_{1}=1-x_{\max _{n}}^{-1}$. In the limit $x_{\max _{n}}>>1$, $L_{\text {res }} \rightarrow k_{p}^{-1} x_{\max _{n}}^{-1 / 2} \simeq 1 / x_{\max _{n}}^{\prime}$ and, hence, the resonance becomes sharper $\left(Q \equiv \lambda_{N n} / L_{\text {res }} \sim x_{\max _{n}}\right)$.

Figure 3 shows a plot of $L_{\text {res }} / c \sim \tau_{\text {opt }}$ versus $\epsilon=$ $E_{z} / E_{\mathrm{WB}}$ for various densities. Notice that, in the regime of high $n_{e 0}$, finite rise-time effects become important at high $\epsilon$, i.e., $\tau_{\text {opt }}$ decreases below $\tau_{\min }$ as $\epsilon$ increases beyond a critical value (e.g., $L_{\text {res }} / c<50$ fs for $\epsilon>0.14$, at $n_{e 0}=10^{16} \mathrm{~cm}^{-3}$ ). Since pulses with $\tau<\tau_{\min }$ cannot be produced, the later pulses in a train are not optimized. Although the later pulses with $\tau=\tau_{\min }>\tau_{\text {opt }}$ will continue to increase $x_{\max }$, they will do this less effectively than a train in which all pulses are of optimal widths. Consequently, although for these later pulses the sensitivity to changes in $\lambda_{N}$ is reduced, a pulse train in this 
high- $n_{e 0}$ regime can be less "amplitude efficient" than a single optimized pulse at the same density, i.e., a greater $I \tau_{\text {tot }}$ is required for the pulse train to achieve a given $E_{z}$ at fixed $n_{e 0}$. High $n_{e 0}$, however, is unfavorable for electron acceleration because of electron-phase detuning, $\Delta W_{\max } \sim E_{z} n_{e 0}^{-3 / 2}$.

Figure 3 indicates that, for low $n_{e 0}$ and up to the previously mentioned critical value of $\epsilon$, the condition $\tau_{\text {opt }} \simeq L_{\text {res }} / c \geq \tau_{\text {min }} \simeq 50$ fs can be satisfied for all of the pulses in a train [as was the case of Fig. 2(b)]. Consequently, multiple sine pulses in this regime are found to be similar to ideal square pulses in that a pulse train is more amplitude efficient than a single pulse at the same density. Specifically, 19 times higher intensity $\left(a_{0}=5.2\right.$ or $\left.I=7.3 \times 10^{19} \mathrm{~W} / \mathrm{cm}^{2}[13]\right)$, corresponding to 6 times more energy $\left(I \tau_{\text {tot }}=32 \mathrm{MJ} / \mathrm{cm}^{2}\right)$, is required of a single pulse $\left(\tau=\tau_{\mathrm{opt}}=450 \mathrm{fs}\right.$ for $\left.n_{e 0}=10^{16} \mathrm{~cm}^{-3}\right)$ to reach the same value of $E_{z}(0.34 \mathrm{GV} / \mathrm{cm})$ as the train of Fig. 2(b). A single pulse with the same intensity and pulse width as the first pulse in Fig. 2(b), corresponding to $2 / 3$ the laser energy $\left(I \tau_{\text {tot }}=3.6 \mathrm{MJ} / \mathrm{cm}^{2}\right)$, results in a 7.7 times smaller $E_{z}(44 \mathrm{MV} / \mathrm{cm})$. Reducing the intensity required to reach large plasma-wave amplitudes also reduces strongly driven instabilities, such as stimulated Raman scattering, self-focusing, or filamentation, which disrupt either the plasma wave or the laser beam. Pulse-to-pulse phase incoherence of the high-frequency laser oscillations can also reduce instabilities.

In order to drive the same $E_{z}$ with the same $I$ as a sine pulse train, a higher $n_{e 0}$ must be used with a single sine pulse. (Recall that, for a single pulse, $E_{z} \sim n_{e 0}^{1 / 2} I$ for $a_{0}^{2}<1$.) Thus, the same value of $E_{z}=0.34 \mathrm{GV} / \mathrm{cm}$ as the train in Fig. 2(b) is obtained by an equivalentintensity single pulse $\left(a_{0}=1.2\right)$ with $\tau=\tau_{\text {opt }}=120 \mathrm{fs}$ at $n_{e 0}=6.4 \times 10^{17} \mathrm{~cm}^{-3}$, and with 13 times less energy $\left(I \tau_{\text {tot }}=480 \mathrm{~kJ} / \mathrm{cm}^{2}\right)$. But, because energy gain favors low $n_{e 0}$, the pulse train in Fig. 2(b) is 510 times more "acceleration efficient," i.e., $\Delta W_{\max }=810 \mathrm{GeV}$ for the pulse train, whereas $\Delta W_{\max }=1.6 \mathrm{GeV}$ for the single pulse. Thus, a pulse train of equivalent intensity-at either equal or lower $n_{e 0}$-is more acceleration efficient than a single pulse [14].

There are several ways of producing the required pulse train in practice. The first is to use Fourier filtering. In this case, a mask is placed in the pulse stretcher of a chirped pulse amplification system [6] to modulate the beam in such a way that, when it is recompressed, a series of pulses with arbitrary spacings and widths will be produced [15]. The minimum rise time of each individ- ual pulse is still governed by the gain bandwidth of the amplifiers. Another possibility is to use several separate compressors with adjustable lengths and delays.

This work was supported by NSF and DOE. The authors acknowledge useful conversations with X. Liu, J. Squier, G. Mourou, J. Krall, A. Ting, P. Sprangle, and W. Mori.

Note added.-We have been recently informed of a paper by S. Dalla and M. Lontano (to be published) on a similar subject.

[1] See, e.g., Advanced Accelerator Concepts, edited by J. S. Wurtele, AIP Conf. Proc. No. 279 (American Institute of Physics, New York, 1993).

[2] T. Tajima and J. M. Dawson, Phys. Rev. Lett. 43, 267 (1979).

[3] L. M. Gorbunov and V. I. Kirsanov, Zh. Eksp. Teor. Fiz. 93, 509 (1987) [Sov. Phys. JETP 66, 290 (1987)]; P. Sprangle et al., Appl. Phys. Lett. 53, 2146 (1988).

[4] C. Clayton et al., Phys. Rev. Lett. 54, 2343 (1985); Y. Kitigawa et al., Phys. Rev. Lett. 68, 48 (1992); F. Amiranoff et al., Phys. Rev. Lett. 68, 3710 (1992); H. Hamster et al., Phys. Rev. Lett. 71, 2725 (1993).

[5] A. I. Akhiezer and R. V. Polovin, Zh. Eksp. Teor. Fiz. 30, 915 (1956) [Sov. Phys. JETP 3, 696 (1956)].

[6] P. Maine et al., IEEE J. Quantum Electron. 24, 398 (1988); G. Mourou and D. Umstadter, Phys. Fluids B 4, 2315 (1992).

[7] P. Sprangle et al., Phys. Rev. Lett. 69, 2200 (1992); J. Krall et al., Phys. Rev. E 48, 2157 (1993); E. Esarey et al., Phys. Fluids B 5, 2690 (1993).

[8] N. E. Andreev et al., Pis'ma Zh. Eksp. Teor. Fiz. 55, 551 (1992) [JETP Lett. 55, 571 (1992)]; T. Antonsen, Jr. and P. Mora, Phys. Rev. Lett. 69, 2204 (1992).

[9] W. P. Leemans et al., Phys. Rev. A 46, 1091 (1992); P. Monot et al., J. Opt. Soc. Am. B 9, 1579 (1992).

[10] V. I. Berezhiani and I. G. Murusidze, Phys. Scr. 45, 87 (1992).

[11] K. Nakajima, Phys. Rev. A 45, 1149 (1992).

[12] E. Esarey et al., IEEE Trans. Plasma Sci. 21, 95 (1993).

[13] Current laser technology has achieved a maximum intensity of $I=5.0 \times 10^{18} \mathrm{~W} / \mathrm{cm}^{2}$.

[14] For square pulses, since $\tau_{\text {opt }}$ increases with $x_{\max }$, the appropriate comparison is at fixed $n_{e 0}$; and, in the low- $n_{e 0}$ regime, square pulses with finite rise times $\tau_{\text {rise }} \simeq \tau_{\min } / 2$ behave as ideal square pulses, provided $\tau_{\text {rise }}<L_{\text {res }} / 2 c$ for all the pulses in the train.

[15] A. M. Weiner et al., IEEE J. Quantum Electron. 28, 908 (1992) 